\title{
Hospitalizações entre crianças e adolescentes no território de abrangência de um serviço de Atenção Primária à Saúde
}

\author{
Hospitalizations among children and adolescents \\ within the scope of a Primary Care Service
}

Maria Lucia Medeiros Lenz ${ }^{1}$ Rui Flores

Norma Vieira Pires ${ }^{2}$

Airton Tetelbom Stein ${ }^{3}$

\section{Resumo}

A hospitalização, além de sofrimento familiar importante, é um evento de custo elevado para o sistema de saúde; além disso, ela pode ser, muitas vezes, prevenida no nível ambulatorial. O objetivo deste estudo foi identificar o percentual de hospitalizações por condições sensíveis à atenção ambulatorial (CSAA) que se referem àquelas em que a atenção efetiva e a tempo podem evitar internação. Foram estudadas 3.329 hospitalizações em menores de 19 anos, ocorridas nos anos de 2001 a 2004, em quatro hospitais do SUS, principais referências para uma população de áreas adscritas de um serviço de Atenção Primária em Saúde (APS). Análises univariadas e multivariadas foram empregadas para verificar associação entre variáveis independentes e a ocorrência de hospitalizações por CSAA. Identificou-se uma taxa anual de hospitalização de 2,9\%. As doenças do aparelho respiratório são o grupo de causa mais freqüente (36\%), seguido do grupo das perinatais (14\%), infecciosas e parasitárias (10\%), do aparelho digestivo $(9 \%)$ e das causas externas $(6 \%)$. As reinternações corresponderam a $16 \%$ do total de internações. A taxa de hospitalização por CSAA foi de 35,6\%, variando de $25 \%$ a $43 \%$ entre as Unidades de APS. As variáveis relacionadas à maior ocorrência de hospitalizações por este motivo foram: idade de um a quatro anos $(\mathrm{p}<0,01)$, ter ido direto para o hospital porque a Unidade estava fechada $(\mathrm{p}=0,04)$ ou pela gravidade do caso $(\mathrm{p}=0,03)$. O estudo indica a necessidade de incrementar ações de vigilância às hospitalizações por CSAA, que ocorrem com maior freqüência nos meses de inverno, às crianças de um a nove anos, por apresentarem-se mais vulneráveis à hospitalização por essas condições, e às reinternações, não reduzidas nos últimos quatro anos.

\begin{abstract}
The hospitalization of a child, in addition to bringing about major family distress, is a costly event for the health system. Besides, it can
\end{abstract}

Palavras-chave: Hospitalização; Atenção Primária à

Saúde; Saúde da Criança; Epidemiologia.
Key Words: Hospitalization; Primary Health

Care; Child Health; Epidemiology.

\footnotetext{
${ }^{1}$ Médicos de Família, Serviço de Saúde Comunitária e especialistas em Saúde Pública, Serviço de Saúde Comunitária do Grupo Hospitalar Conceição, Porto Alegre, Rio Grande do Sul, Brasil.

${ }_{3}^{2}$ Enfermeira especialista em Saúde Pública, Serviço de Saúde Comunitária do Grupo Hospitalar Conceição, Porto Alegre, Rio Grande do Sul, Brasil. ${ }^{3}$ Médico de Família e Comunidade e doutor em Epidemiologia da Gerência de Ensino e Pesquisa, Grupo Hospitalar Conceição, Porto Alegre, Rio Grande do Sul, Brasil.
} 
often be avoided on an outpatient care basis. The aim of this study was to identify the percentage of hospitalizations for Ambulatory Care Sensitive (ACS) conditions, i.e. those for which effective and timely care can avoid hospital admission. We investigated 3.329 hospitalizations of patients under 19 years of age, occurred between 2001 and 2004 in four hospitals of the Brazilian Health System (SUS), the main references for a population of areas astricted to a primary care service (PCS). The hospitalizations in this population were identified using the hospital information system of the GHC (Grupo Hospitalar Conceição). Univariate and multivariate analysis were employed to verify associations between independent variables and the occurrence of admissions for ACS conditions. A hospitalization rate of $2.9 \%$ was found for the studied age group. Respiratory system disorders were the leading cause of admission (36\%), followed by perinatal diseases (14\%), infectious and parasitic diseases (10\%), disorders of the digestive system (9\%), and external causes (6\%). Readmission accounted for $16 \%$ of the total admissions. The bospitalization rate for ACS conditions was 35.6\%, ranging from $25 \%$ to $43 \%$ across the Health Care Centers of the PCS. The variables associated with higher occurrence of hospitalizations for this reason were: age between 1 and 4 years $(p<0.01)$; having gone straight to the hospital because the Unit was closed ( $p=0.04)$; and having gone straight to the hospital due to the severity of the case $(p=0.03)$. The study points to the need for a better monitoring of hospitalizations for ACS conditions - occurring more frequently in the winter months and involving children from 1 to 9 years of age for being more vulnerable to respiratory diseases - and of the number of readmissions, which did not decrease over the last four years.

\section{Introdução}

Os serviços de Atenção Primária à Saúde (APS) apresentam, entre outras características, facilidade de acesso à população, integralidade, longitudinalidade no cuidado, qualidade da atenção e coordenação das ações, dentro do sistema de saúde. As ações de promoção e prevenção à saúde de populações de áreas delimitadas são enfatizadas por esses serviços ${ }^{1,2,3}$.

A hospitalização, além de sofrimento familiar importante, é um evento de custo elevado e, muitas vezes, pode ser prevenida com ações em nível ambulatorial. Sendo assim, as freqüências de internação e reinternação são consideradas importantes indicadores para avaliação dos serviços de APS $^{1}$.

Estudos têm sido realizados no sentido de identificar situações de risco para a hospitalização ${ }^{1,4,5,6,7,8,9,10,11,12,13}$.

Dentre os motivos de hospitalização, encontramos condições consideradas sensíveis à atenção ambulatorial (CSAA) e referem-se àquelas em que a atenção ambulatorial efetiva e a tempo podem evitar internações: prevenindo enfermidades, tratando precocemente a enfermidade aguda ou controlando a enfermidade crônica ${ }^{4,7,14,15,16,17}$.

A freqüência de internações por CSAA é um indicador importante da qualidade da assistência à saúde $\mathrm{e}^{1,4,5,6,7}$ e relaciona-se fortemente com o acesso aos serviços ${ }^{14,15,18}$. Considera-se, ainda, que uma oferta universal de APS inserida em um sistema de saúde fortemente integrado é fator relevante para evitar internações por $\operatorname{CSAA}^{19}$.

Estabelecendo ações de vigilância à saúde, tal como o projeto De Volta pra Casa, o Serviço de Saúde Comunitária identifica situações de risco, como a hospitalização de crianças e adolescentes e o acompanhamento adequado de suas famílias pelas equipes ${ }^{20}$.

O objetivo deste trabalho foi de descrever os motivos de hospitalizações entre crianças e adolescentes pertencentes ao território de abrangência do SSC e identificar a freqüência de internações por CSAA, assim como as tendências dessas internações de 2001 a 2004. Procurou-se ainda avaliar os serviços de saúde utilizados anteriormente à hospitalização, a existência de vínculo com o serviço de Atenção Primária à Saúde e o tempo decorrido entre a hospitalização e o contato com a equipe de saúde.

\section{Material e métodos}

O GHC está vinculado ao Ministério da Saúde e é composto por quatro hospitais e pelo SSC, sendo este responsável por uma população de 84.994 moradores de áreas adscritas da zona norte da cidade de Porto Alegre (RS). A população de 0 a 19 anos (incompletos) é de 28.602, sendo $51 \%$ do gênero masculino ${ }^{20}$.

O SSC é constituído por 12 Unidades de Saúde, 
compostas por equipes multiprofissionais que desenvolvem assistência, formação de recursos humanos para o SUS e novas tecnologias em Atenção Primária. O horário de funcionamento das Unidades é de segunda a sexta, das 8 às 18 horas.

Foram incluídos no estudo usuários de 0 a 19 anos (incompletos), moradores das áreas de adscrição das 12 Unidades do SSC que permaneceram em um dos quatro hospitais do GHC por um período superior a 24 horas.

Foram excluídas do estudo as internações não comprovadas pelas Equipes de Saúde como sendo da área adscrita, as internações de usuários que se mudaram dessa área durante o período do estudo e as internações de usuários com endereços não localizados pelas equipes.

O delineamento realizado neste estudo foi transversal e todos os casos de internação ocorridos no período de $1^{\circ}$ de janeiro de 2001 a 31 de dezembro de 2001 foram estudados.

O processo de coleta de dados é parte de ações sistemáticas de um Programa de Vigilância à Saúde de morbidade hospitalar do SSC, em funcionamento desde 2000, e conhecido como De Volta pra Casa.

O Prontuário Eletrônico utilizado no GHC possibilita a identificação diária dos usuários moradores no território do SSC, suas idades e as datas e motivos das internações. Estas informações foram registradas em um instrumento de coleta e também digitadas em um banco de dados específico.

As equipes, ao receberem a informação da internação, providenciam contato com as famílias, comprovando ou não a moradia na área de adscrição, coletando informações referentes à utilização de serviços de saúde e complementando o instrumento de coleta.

O diagnóstico definitivo no momento da alta, coletado com nova pesquisa no Prontuário Eletrônico, foi acrescido no banco de dados.

As variáveis estudadas foram: gênero (masculino ou feminino), datas de nascimento e de hospitalização (dia, mês e ano), número de registro no hospital (número de identificação do usuário no GHC), diagnóstico principal da alta $^{22}$, unidade de saúde de referência (número da Unidade), presença de prontuário família na Unidade (sim ou não), caminho percorrido anterior à hospitalização (encaminhado por profissional da Unidade, encaminhado por outro serviço, ter ido direto ao hospital por Unidade fechada, não ter conseguido consulta ou pela gravidade do caso), data da alta hospitalar, data do contato entre equipe e família após internação e local de acompanhamento após alta (na Unidade do SSC ou não).

A freqüência de internação foi calculada relacionando o número de internações e a população total das áreas de abrangência nas faixas etárias de 0 a 19 anos. A freqüência de reinternação foi calculada pela razão dos números de registros que apareceram mais de uma vez e o total de hospitalizações no mesmo período. As hospitalizações por CSAA foram identificadas conforme Quadro 1. A freqüência de internações por esses motivos foi calculada pela razão do número de eventos ocorridos pelo total de internações. A sazonalidade das internações foi identificada pela data de internação. O tempo de hospitalização foi calculado a partir das datas de internação e de alta. O tempo decorrido entre a hospitalização e o contato com a família foi calculado a partir da data da internação e data do contato. A presença de vínculo prévio entre família e equipe de saúde foi avaliada por meio da existência de número de prontuário na Unidade. O caminho percorrido nos serviços de saúde, anterior à hospitalização, foi avaliado da seguinte forma: encaminhado pela Unidade, direto para o hospital (US fechada), direto para o hospital (gravidade do caso) e direto para o hospital (não conseguiu consulta).

A análise dos dados foi realizada em Epi-Info versão 6.1 e SPSS versão 12. Utilizou-se estatística descritiva e de inferência com média e desvio padrão para a variável idade e percentual para as variáveis categóricas. Teste t-student foi utilizado para comparar a variável contínua e o teste qui-quadrado para variáveis categóricas. O valor de $\mathrm{p}<0,05$ foi considerado estatisticamente significativo.

Foi procedida a análise descritiva mediante o cálculo de médias e proporções. Para estudar a associação entre internação por CSAA e fatores de interesse, foi realizada a 
análise bivariada e foram calculadas as razões de chances (OR) e seus respectivos intervalos de confiança de 95\% (IC95\%). Com a finalidade de estudar o efeito independente dos diversos fatores, foi realizada uma análise multivariada, mediante a utilização de regressão logística não-condicional, calculando-se, desta forma, as Razões de Chances e os respectivos IC95\%.

\section{Resultados}

O total de hospitalizações entre 2001 e 2004 foi de 4.003. Excluíram-se 293 (7,3\%) que correspondiam a moradores fora da área de atuação, 225 (5,6\%) por mudança de endereço e 156 (3,9\%) em que os endereços não foram localizados pela equipe. $\mathrm{O}$ tamanho da amostra foi de 3.329 hospitalizações.

Não houve variação na freqüência de internação da população adscrita do SSC de 2001 a 2004 nos hospitais do GHC. Considerando a população menor de 19 anos do SSC, calculou-se uma taxa anual de hospitalização de $2,9 \%$. As reinternações corresponderam a $16 \%$ do total de internações ocorridas e também não apresentaram variação nos quatro anos de estudo $(\mathrm{p}=0,11)$.

Os meninos hospitalizam-se mais que as meninas em todas as faixas etárias $(p<0,001)$, sendo que a diferença torna-se maior a partir dos quatro anos de idade. A freqüência de hospitalização foi inversamente proporcional à idade da criança.

A mediana do tempo de internação foi três dias, e $98 \%$ das crianças não permanecem no hospital por um período superior a 30 dias.

Identificamos que $94,2 \%$ das crianças e adolescentes moradores das áreas de atuação do SSC e que se hospitalizaram no GHC têm prontuário nas Unidades do SSC.

Em relação ao caminho percorrido até o hospital, identificou-se que $24 \%$ foram encaminhados por profissional da Unidade do SSC, 3,8\% foram encaminhados por profissional de outro serviço, $24,2 \%$ foram direto porque a Unidade encontrava-se fechada, 1,9\% foram direto porque não conseguiram consulta, 25,4\% foram direto pela gravidade do caso e $20,6 \%$ por outra situação, como por exemplo, hospitalizarem-se logo após o nascimento.

O primeiro contato após internação ocorreu em um tempo mediano de 13 dias. Em relação ao local de acompanhamento após alta, identificou-se que $81,8 \%$ das crianças serão acompanhadas nas Unidades do SSC e 15\% em outros serviços.

Considerando os principais motivos de internação em capítulos do CID 10, observamos: doenças respiratórias (36\%), afecções perinatais (14\%), doenças infecciosas e parasitárias $(10 \%)$, doenças digestivas $(9 \%)$, lesões, envenenamentos e outras causas externas $(6 \%)$.

Conforme Tabela 1, os dez primeiros diagnósticos principais representam $50 \%$ do total de internações ocorridas no período do estudo. Observou-se uma redução de internação por septicemia $(p=0,01)$ e epilepsia $(p=0,02)$ e aumento das hospitalizações por bronquiolite $(\mathrm{p}=0,01)$ ao longo destes quatro anos.

Observamos um aumento estatisticamente significativo na freqüência do total de hospitalizações $(p<0,01)$ e de hospitalizações por CSAA $(p<0,01)$ nos meses de maio, junho e julho (Figura 1).

O total de internações por CSAA foi de 1.185, representando uma freqüência de 35,6\% (variando de $25 \%$ a 43\% entre as US) e não apresentando variação ao longo destes quatro anos $(\mathrm{p}=0,5)$. Identificaram-se hospitalizações por doenças imunopreveníveis e parasitárias não relacionadas na tabela 1, tais como hepatites A e B, rubéola, caxumba e ascaridíase.

Conforme a Tabela 2, após análise ajustada, meninos e meninas não apresentam diferença estatística em hospitalização por CSAA (OR=1,0 - IC 95\% 0,9 a 1,2). Crianças de um a quatro e de cinco a nove anos apresentam três e duas vezes mais chances, respectivamente, para hospitalizar por CSAA (OR=3,1-IC95\% 2,6 a 3,7 e OR=2,1- IC95\% 1,7 a 2,6) em relação aos menores de um ano.

Pacientes que foram para o hospital devido à US encontrar-se fechada, ou pela gravidade do caso, apresentaram 1,3 e 1,4, respectivamente, mais chances de hospitaliza- 
rem-se por CSAA.

\section{Discussão}

Estudos de morbidade são importantes no controle das doenças e no planejamento de ações ${ }^{5,6,11,23,24}$. Além disso, captam aspectos do processo saúde-doença que escapam das estatísticas de mortalidade ${ }^{11}$.

A taxa anual de hospitalização na população de 0 a 19 anos do território de abrangência do SSC foi de 2,9\%. Embora avaliações realizadas no SSC evidenciem que o GHC é referência para $70 \%$ desta população ${ }^{25}$, existe uma limitação no estudo devido ao fato de que não foram estudadas as hospitalizações ocorridas fora do GHC, devido à inacessibilidade das informações. Esta limitação se estende para comparações com taxas de hospitalização encontradas em outros estudos de base populacional ${ }^{11,10,24,26}$.

A freqüência de reinternação de $16 \%$ foi considerada alta e semelhante à encontrada em outros estudos brasileiros na mesma faixa etária ${ }^{27,28}$. No Chile, um estudo considerou muito alta uma taxa de reinternação de 9,5\%, embora tenha sido avaliada no primeiro mês após alta hospitalar ${ }^{29}$.

Os meninos hospitalizaram-se mais que as meninas em todas as faixas etárias, assim como observado em outros estudos ${ }^{11,13}$. As crianças, quanto menores, apresentaram-se mais vulneráveis à hospitalização. Um estudo calcula que, a cada ano de vida, as crianças diminuem em 13\% as chances de se hospitalizar ${ }^{13}$. As crianças, quanto menores, apresentam maior impacto negativo à saúde decorrente deste evento ${ }^{24}$.

O tempo mediano de três dias de permanência no hospital pode ser considerado baixo e semelhante ao encontrado em outro estudo brasileiro ${ }^{28}$, uma vez que o tempo previsto pelo Ministério da Saúde para a hospitalização de crianças é de cinco dias ${ }^{13}$.

A maior parte das crianças ou adolescentes moradores das áreas de atuação do SSC que se hospitalizou apresenta prontuário nas Unidades do SSC. Quando os pacientes foram encaminhados ao hospital por um serviço de saúde, a maioria foi encaminhada por profissional das Unidades do SSC. Quando foram direto ao hospital, muito poucos foram por não ter conseguido consulta. Estas informações evidenciam que o SSC é a principal referência em APS para essa população e encontra-se acessível durante o período de funcionamento. Refletem, ainda, bons indicadores na qualidade da atenção, uma vez que são utilizados na avaliação de acesso, longitudinalidade e na coordenação dos serviços de APS ${ }^{1}$.

A coordenação da atenção também pode ser medida por meio da existência de um sistema de informação que permite realizar Vigilância à Saúde, identificando a passagem e efetividade da atenção aos pacientes em outros pontos do sistema de saúde, como ambulatórios especializados e internação. Após ter acesso à informação da internação da criança ou do adolescente, as equipes do SSC entram em contato com a família em um tempo adequado, completando a vigilância e possibilitando uma assistência integral.

Os cinco principais grupos de causas de internação entre 0 a 19 anos no SSC são os mesmos encontrados em outros estudos brasileiros ${ }^{29}$. No entanto, o percentual de internações por doenças respiratórias é maior no SSC (36\%) do que para o país (25\%), o que poderá estar relacionado às temperaturas médias mais baixas ${ }^{30}$.

A asma é o principal motivo de hospitalização no SSC e sua freqüência é semelhante às encontradas em outros estudos ${ }^{6,15,16,31}$. Estudo identifica fatores de risco para internação por este motivo: início precoce da doença, baixa escolaridade materna, gravidade da doença e duas ou mais consultas de emergência por mês ${ }^{31}$.

Ao longo de 2001 a 2004, houve aumento no número de internações por bronquiolite. Estudo recente demonstra que a amamentação exclusiva é importante fator de proteção para evitar internações por este motivo. A criança com período de amamentação inferior a um mês tem sete vezes mais risco para hospitalização por bronquiolite nos primeiros três meses de vida ${ }^{8}$.

A redução do número de internações por epilepsia poderá estar relacionada a um trabalho integrado com o ambulatório especializado, onde foram realizadas ações, como: avaliação do especialista de todas as crianças que vinham sendo hospitalizadas por este motivo, educação con- 
tinuada para profissionais do SSC e elaboração de material instrucional fornecido às Equipes de Saúde.

Em relação aos motivos de hospitalização, fato interessante para o planejamento de estratégias de educação permanente na construção de diretrizes clínicas e na educação e saúde é o de que os dez diagnósticos principais correspondem à metade das hospitalizações ocorridas no período do estudo.

A freqüência de internação por CSAA, no presente estudo, de 35,6\% foi semelhante à de 33\% encontrada em estudo americano ${ }^{26}$. No entanto, este percentual variou de 25\% a 43\% entre as diferentes Unidades do SSC, evidenciando a possibilidade de diferenças na qualidade de atenção. Uma melhor avaliação dos motivos dessas diferenças esteve limitada devido à ausência de variáveis socioeconômicas no sistema de informação, determinantes de hospitalização por CSAA. A faixa etária de maior risco para internação por CSAA é a de um a quatro anos, reforçando a necessidade de manter a vigilância à saúde dessas crianças de forma sistematizada até os cinco anos de idade.

Outra limitação do estudo é a possível superestimação do OR em relação a RP, o qual foi utilizado para calcular medidas de associação ${ }^{32}$. No entanto, com freqüências inferiores a $10 \%$, a diferença entre essas medidas de associação não é significativa.

$\mathrm{O}$ indicativo de ter passado anteriormente nas unidades de saúde foi fator de proteção para internação por CSAA. Famílias que foram direto para o hospital ou porque as Unidades estavam fechadas, ou por referirem gravidade da situação, estiveram relacionadas a maiores freqüências de hospitalizações por CSAA. Essa informação leva a pensar que um planejamento ampliando o horário das unidades e enfatizando educação em saúde poderia reduzir internações por estes motivos.

\section{Conclusões}

O estudo aponta para a importância em destinar esforços na redução de hospitalizações por CSSA no SSC, principalmente as que se referem às doenças respiratórias que ocorrem com maior freqüência nos meses de inverno.
Estudo recente evidencia que, se por um lado, a maior disponibilidade de leitos poderia propiciar internações desnecessárias, essa sobreutilização poderia também estar relacionada a problemas de acesso e qualidade da atenção ambulatorial ${ }^{33}$.

A maior parte da população estudada utiliza o SSC, tem prontuário nas Unidades, poucos não conseguiram consulta anteriormente à hospitalização, a equipe é informada prontamente, está organizada para contato precoce com a família, conta com sistema de informação que permite avaliação contínua, mas apresenta variação entre as Unidades na taxa de hospitalização por CSAA. Assim, um melhor desempenho não somente na questão do acesso, mas também na qualidade da atenção e efetividade das consultas no SSC, têm importância no sentido de evitar hospitalizações desnecessárias no SSC.

Ações mais efetivas poderiam ser planejadas a partir de um novo estudo que controlasse as diferenças socioeconômicas. As iniqüidades existentes poderiam ser relacionadas mais diretamente ao SSC. Sugere-se com este objetivo acrescentar variáveis como renda da família e escolaridade da mãe no sistema de informação específico das hospitalizações.

Os resultados apontados recomendam priorizar a vigilância às CSAA, incluindo neste grupo situações encontradas no presente estudo e não descritas no Quadro 1, às reinternações, sem redução nestes quatro anos.

Conhecer informações sobre morbidade em territórios adscritos de APS possibilita novos subsídios para o planejamento de ações de educação permanente dos profissionais, elaboração de diretrizes clínicas e educação em saúde. Avaliar continuamente os serviços é fundamental na sensibilização de gestores e profissionais para uma atenção qualificada e no incentivo da população para desenvolver o controle social. 
Quadro 1. Condições sensíveis de atenção ambulatorial (CSAA)*

\begin{tabular}{|c|c|c|c|}
\hline CID 10 & Doença & CID 10 & Doença \\
\hline $\begin{array}{l}\text { A } 37 \\
\text { A33, A34, A35 } \\
\text { A } 80 \\
\text { G00.0 } \\
\text { I00 } \\
\text { I01 }\end{array}$ & $\begin{array}{l}\text { Coqueluche } \\
\text { Tétano } \\
\text { Poliomielite } \\
\text { Meningite haemophilus } \\
\text { Febre reumática } \\
\text { Febre reumática com } \\
\text { compr. cardíaco }\end{array}$ & $\begin{array}{l}\mathrm{I} 24 \\
\mathrm{I} 25 \\
\mathrm{I} 20\end{array}$ & $\begin{array}{l}\text { Outras formas agudas e subagudas } \\
\text { de doença isquêmica } \\
\text { Angina do peito }\end{array}$ \\
\hline $\begin{array}{l}\text { G40 } \\
\text { R56 }\end{array}$ & $\begin{array}{l}\text { Epilepsia } \\
\text { Convulsões }\end{array}$ & $\begin{array}{l}\text { L00, L01 } \\
\text { L02, L03 } \\
\text { L04, L08 }\end{array}$ & $\begin{array}{l}\text { Celulite / abcesso dedos mãos e pés } \\
\text { Outras localizações de } \\
\text { celulites / abcessos } \\
\text { Linfadenite aguda } \\
\text { Outras infecções de pele e tecido } \\
\text { celular subc. }\end{array}$ \\
\hline $\begin{array}{l}\text { H66 } \\
\text { J02 } \\
\text { J03 } \\
\text { J06 } \\
\text { J31.2 }\end{array}$ & $\begin{array}{l}\text { Otite média sup. e NE } \\
\text { Faringite aguda } \\
\text { Amigdalite aguda } \\
\text { IVAS localização NE } \\
\text { Faringite crônica }\end{array}$ & $\begin{array}{l}\text { E10 } \\
\text { E11 } \\
\text { E12 } \\
\text { E14 }\end{array}$ & $\begin{array}{l}\text { Diabetes mellitus sem complicação } \\
\text { DM / cetoacidose } \\
\text { DM / come } \\
\text { DM / com alterações renais } \\
\text { DM / com complicações NE }\end{array}$ \\
\hline $\begin{array}{l}\text { A16, A17,A18, } \\
\text { A19 }\end{array}$ & $\begin{array}{l}\text { Qualquer tuberculose, exceto } \\
\text { tbc primária }\end{array}$ & $\begin{array}{l}\text { E15 } \\
\text { E16 } \\
\text { K52 }\end{array}$ & $\begin{array}{l}\text { Hipoglicemia NE } \\
\text { Outras gastroenterites não- } \\
\text { infecciosas }\end{array}$ \\
\hline A50 & Sífilis congênita & $\begin{array}{l}\text { N15.8 } \\
\text { N10, N11 } \\
\text { N12, } \\
\text { N15, } \\
\text { N28.8 }\end{array}$ & $\begin{array}{l}\text { Infecções do rim } \\
\text { ITU de local NE } \\
\text { Outras afecções da uretra e T.U. NE }\end{array}$ \\
\hline $\begin{array}{l}\text { J41 } \\
\text { J43 } \\
\text { J47 } \\
\text { J44 } \\
\text { J20, J40 }\end{array}$ & $\begin{array}{l}\text { Bronquite crônica } \\
\text { Enfisema } \\
\text { Bronquiectasia } \\
\text { Obst. Crônica vias resp. NE } \\
\text { Bronquite aguda }\end{array}$ & $\begin{array}{l}\text { K02 } \\
\text { K03 } \\
\text { K05 } \\
\text { K08 } \\
\text { K12 }\end{array}$ & $\begin{array}{l}\text { Doenças do tecido dentário duro } \\
\text { Doenças da polpa e tecido periapical } \\
\text { Doenças gengivais } \\
\text { Outras doenças de afecção dos } \\
\text { dentes e estruturas } \\
\text { Doenças dos tecidos moles da boca } \\
\text { exceto lesões da gengiva e língua }\end{array}$ \\
\hline $\begin{array}{l}\text { J13 } \\
\text { J14 } \\
\text { J13 } \\
\text { J16, J18 } \\
\text { J15 } \\
\text { J18 } \\
\text { J18 }\end{array}$ & $\begin{array}{l}\text { Pneumonia pneumocócica } \\
\text { Pneumonia p/ haemophilus } \\
\text { Pneumonia p/ streptococos } \\
\text { Pneumonia bacteriana NE } \\
\text { Pneumonia devido a outros } \\
\text { Broncopneumonia NE } \\
\text { Pneumonia microorg. NE }\end{array}$ & E86 & Depleção de volume líquido \\
\hline J45 & Asma & D50 & Anemia por deficiência de ferro \\
\hline $\begin{array}{l}\text { I50 } \\
\text { I11 } \\
\text { J81 }\end{array}$ & $\begin{array}{l}\text { Insuficiência cardíaca } \\
\text { Doença cardíaca hipert. } \\
\text { Edema agudo de pulmão }\end{array}$ & $\begin{array}{l}\text { E40 } \\
\text { E41, E42 } \\
\text { E43, E44 } \\
\text { E55 } \\
\text { E64.3 }\end{array}$ & $\begin{array}{l}\text { Kwashiorkor } \\
\text { Marasmo nutricional } \\
\text { Outras formas de desnutrição } \\
\text { Raquitismo } \\
\text { Efeitos tardios do raquitismo }\end{array}$ \\
\hline $\begin{array}{l}\mathrm{I} 10 \\
\mathrm{I} 10 \\
\mathrm{I} 11\end{array}$ & $\begin{array}{l}\text { Hipertensão E maligna } \\
\text { Hipertensão Essencial NE } \\
\text { D cardíaca hipertensiva }\end{array}$ & R62 & $\begin{array}{l}\text { Falha no desenvolvimento } \\
\text { fisiológico esperado }\end{array}$ \\
\hline
\end{tabular}

* Atualizado para CID 10 e traduq̨ido para o Português. Fonte: Casanova \& Starfield,5. 
Tabela 1. Distribuição dos motivos de internação em um dos quatro hospitais do GHC, por ano, no território de abrangência do SSC de 2001 a 2004. GHC, SSC 2005.

\begin{tabular}{|c|c|c|c|c|c|}
\hline $\begin{array}{l}\text { Diagnóstico Principal } \\
\text { na Hospitalização }\end{array}$ & $\begin{array}{c}2001 \\
n=826 \\
\text { f ( } \%)\end{array}$ & $\begin{array}{c}2002 \\
\mathrm{n}=807 \\
\mathrm{f}(\%)\end{array}$ & $\begin{array}{c}2003 \\
\mathrm{n}=842 \\
\mathrm{f}(\%)\end{array}$ & $\begin{array}{c}2004 \\
\mathrm{n}=854 \\
\mathrm{f}(\%)\end{array}$ & $\begin{array}{c}\text { Total } \\
\mathrm{n}=3.329 \\
\mathrm{f}(\%)\end{array}$ \\
\hline $1^{\text {a }}$ Asma Brônquica* & $131(16)$ & $117(14)$ & $158(19)$ & $140(16)$ & $546(16)$ \\
\hline $2^{\mathrm{a}}$ Bronquiolite & $30(4)$ & $67(8)$ & $64(8)$ & $65(8)$ & $226(7)$ \\
\hline $3^{\text {a }}$ Gastroenterites* & $58(7)$ & $52(6)$ & $70(8)$ & $46(5)$ & $226(7)$ \\
\hline $4^{a}$ Pneumonia* & $48(6)$ & $31(4)$ & $36(4)$ & $39(5)$ & $154(5)$ \\
\hline $5^{a}$ Sinusites & $36(4)$ & $23(3)$ & $29(3)$ & $22(3)$ & $110(3)$ \\
\hline $6^{\text {a }}$ Prematuridade & $24(3)$ & $26(3)$ & $30(4)$ & $26(3)$ & $106(3)$ \\
\hline $7^{a}$ Icterícia neonatal & $20(2)$ & $22(3)$ & $17(2)$ & $30(4)$ & $89(3)$ \\
\hline $8^{a}$ Epilepsia* & $20(2)$ & $31(4)$ & $18(2)$ & $13(2)$ & $82(2)$ \\
\hline $9^{\text {a }}$ Septicemia & $27(3)$ & $13(2)$ & $10(1)$ & $14(2)$ & $64(2)$ \\
\hline $10^{\mathrm{a}}$ Desidratação* & $17(2)$ & $15(2)$ & $11(1)$ & $10(1)$ & $53(2)$ \\
\hline Total & $411(50)$ & $397(49)$ & $443(53)$ & $405(47)$ & $1.656(50)$ \\
\hline
\end{tabular}

* Condiçoes Sensiveis de Atenção Ambulatorial (CSAA)

Figura 1. Distribuição do total das hospitalizações e das hospitalizações por CSAA nos 12 meses do ano. GHC, SSC 2005.
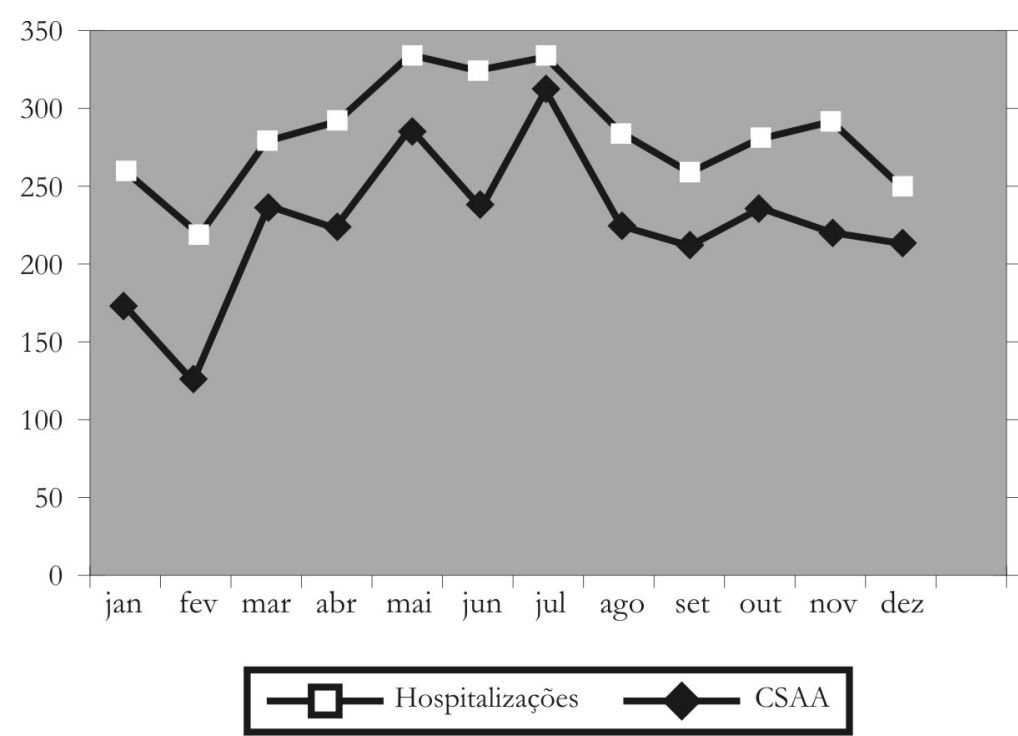
Tabela 2. Fatores associados à ocorrência de hospitalização por condição sensível à atenção ambulatorial, considerando as análises bivariada e de regressão logística múltipla, apresentando estimativas de odds ratio e intervalo de confiança de $95 \%$.

GHC, SSC 2005.

\begin{tabular}{|c|c|c|c|c|c|c|c|}
\hline & \multicolumn{2}{|c|}{ CSAA } & \multicolumn{2}{|c|}{$\begin{array}{c}\text { Análise } \\
\text { Bivariana }\end{array}$} & \multicolumn{3}{|c|}{$\begin{array}{l}\text { Regressão } \\
\text { Logística }\end{array}$} \\
\hline & $\mathbf{n}$ & $\mathrm{f}(\%)$ & OR & IC $95 \%$ & OR & IC $95 \%$ & $\mathrm{p}$ \\
\hline \multicolumn{8}{|l|}{ Gênero } \\
\hline Feminino & 1.888 & $509(35)$ & 1,0 & - & 1,0 & - & - \\
\hline Masculino & 1.438 & $674(36)$ & 1,0 & 0,9 a 1,2 & 1,0 & 0,9 a 1,2 & 0,61 \\
\hline \multicolumn{8}{|l|}{ Faixa Etária / Anos } \\
\hline$<1$ & 1.437 & $378(26)$ & 1,0 & - & 1,0 & - & - \\
\hline 1 a 4 & 962 & $517(54)$ & 3,2 & 2,7 a 3,9 & 3,1 & 2,6 a 3,7 & $<0,01$ \\
\hline 5 a 9 & 519 & $228(44)$ & 2,2 & 1,8 a 2,7 & 2,1 & 1,7 a 2,6 & $<0,01$ \\
\hline 10 a 19 & 411 & $62(15)$ & 0,5 & 0,4 a 0,7 & 0,5 & 0,4 a 0,6 & $<0,01$ \\
\hline \multicolumn{8}{|l|}{$\begin{array}{l}\text { Utilização do Serviço APS } \\
\text { Prontuário na US do SSC: }\end{array}$} \\
\hline Com prontuário na US & 2.556 & $948(37)$ & 1,0 & - & 1,0 & - & - \\
\hline Sem prontuário na US & 157 & $37(24)$ & 0,5 & 0,4 a 0,8 & 0,7 & 0,4 a 0,9 & 0,01 \\
\hline \multicolumn{8}{|l|}{ Caminho percorrido até o hospital: } \\
\hline Encaminhado pela US & 559 & $197(35)$ & 1,0 & - & 1,0 & - & - \\
\hline Direto para o hospital (US fechada) & 563 & $253(45)$ & 0,7 & 0,5 a 0,9 & 1,3 & 1,0 a 1,7 & 0,04 \\
\hline Direto para o hospital (gravidade do caso) & 593 & $253(43)$ & 0,7 & 0,6 a 0,9 & 1,4 & 1,0 a 1,7 & 0,03 \\
\hline
\end{tabular}

\section{Agradecimentos}

Agradecemos às Equipes de Saúde e a todas as mães e crianças moradoras das áreas de atuação do SSC que tornaram possível a realização deste estudo.

\section{Referências}

1. Starfield B. Primary Care. Concepts, Evolution and Policy. New York: Oxford University Press; 1992.

2. Brasil. Ministério da Saúde. Programa de Saúde da Família. Brasília (DF): Departamento de Atenção Básica, Ministério da Saúde; 2004.

3. Harzhein E, Stein AT, Álvarez-Dardet C. et al. Revisão Sistemática sobre aspectos metodológicos das pesquisas em atenção primária no Brasil. Revista da AMRIGS. 2005; 49 (4): 248-252.

4. Billings J, Anderson G, Newman L. Recent findings on preventable hospitalizations. Health Aff. 1996; (15): 239-49. 5. Silva AAM, Gomes UA, Tonial SR, Silva RA. Fatores de risco para hospitalização de crianças de um a quatro anos em
São Luís, Maranhão, Brasil. Cad. Saúde Pública. 1999; 15(4). 6. Casanova C, Starfield B. Hospitalizations of children and access to primary care: A crossnational comparison. Int J Health Serv, v.25, n.2, p.283-94,1995.

7.Casanova, C., Starfield, B. Pediatric Hospitalization due to ambulatory care sensitive conditions in Valencia (Spain). Int J Health Care. 1996; 8(1): 51-59.

8. Albernaz EP, Menezes AB, César J. et al. Hospitalização por bronquiolite aguda como fator de risco para sibilância recorrente. Cad. Saúde Pública. 2000; 16(4):1049-1057.

9. Barros F, Victora C. Epidemiologia da saúde Infantil. 3 ed. São Paulo: Hucitec - Unicef; 1998.

10. Caetano JRM. et al. Fatores Associados à internação hospitalar de crianças menores de cinco anos. Revista Saúde Pública. 2002; 36(3).

11. Cesar J. et al. Hospitalizações em menores de um ano pertencentes a duas coortes de base populacional no sul do Brasil: tendências e diferenciais. Cad. Saúde Pública. 1996;12 (supl.1). 
12. Perrin JM. et al. Variations in rates of hospitalization of children in three urban communities. New England Journal of Medicine. 1989; 320 (18):1183-1187.

13. Castro MS, Carvalho MS, Travassos C. Fatores Associados às Readmissões Hospitalares em um Hospital Geral Brasileiro. Cadernos de Saúde Pública. 2005; 21(4). 14. Marquez-Caldeirón S. et al. Factores asociados a la hospitalización por procesos sensibles a cuidados ambulatorios en los municipios. Gaceta Sanitaria. 2003; 17(5): 360-367.

15. Bermudez - Tamayo C. et al. Organizational characteristics of primary care and hospitalization for the main ambulatory care sensitive conditions. Atenção Primaria. 2004; 15(6):305-11.

16. Weissman J, Gatsonis C, Epstein A. Rates of avoidable hospitalization by insurance status in Massachutsetts and Maryland. JAMA. 1992; (268): 2388-94.

17. Gadomski A, Jenkins P, Nichols M. Impact of medicaid primary care provider and preventive care on pediatric hospitalization. Pediatrics. 1998; 101(3).

18. Parchman M, Culler S. Primary care physicians and avoidable hospitalizations. J Fam Pract. 1994; 39(2):123-8. 19. Mendes EV. Os Grandes Dilemas do SUS. Tomo II. Salvador (BA): Casa da Qualidade; 2001.

20. Lenz ML, Pires N, Takeda S, Flores R. Vigilância à Saúde da Criança e Adolescente: - De volta pra casa. Núcleo de Epidemiologia. Serviço de Saúde Comunitária do Grupo Hospitalar Conceição. Ações Premiadas no VI concurso de Inovações na Gestão Pública Federal. ENAP; 2001.

21.Instituto Brasileiro de Geografia e Estatística. Censo 2000. Disponível em: http://www.ibge.gov.br

22. Organização Mundial de Saúde. Classificação Estatística Internacional de Doenças e Problemas Relacionados à Saúde. [Décima revisão]. São Paulo: Universidade de São Paulo; 1997. v1.

23. Stein AT. Acesso a atendimento médico continuado: uma estratégia para diminuir consultas não urgentes em serviços de emergência. [Tese]. Porto Alegre (RS): Universidade Federal do Rio Grande do Sul; 1998.

24. Cesar J. et al. Utilização de serviços de saúde por menores de cinco anos no extremo sul do Brasil. Cad. Saúde Pública. 2002; 18(1).

25. Serviços de Saúde Comunitária. Relatório de Avaliação: Ações Materno-Infantis. [s.l.:s.n.]; 2004. (mimeo).

26. Parker J, Schoendorf K. Variation in hospital discharges for ambulatory care-sensitive conditions among children. Pediatrics. 2000; 106(4).

27. Madeira LM. et al. Reinternação Pediátrica: Conhecendo a Magnitude do Problema. Revista Brasileira de Enfermagem. 1996; 49(4):531-548.

28. Rocha JS, Simões BJG, Gudes GLM. Assistência Hospitalar como Indicador de Desigualdade Social. Revista de saúde Pública. 1997; 31(5).

29. Brasil. Ministério da Saúde. Sistema de Informações Hospitalares do SUS - SIH/SUS. Disponível em: http:// tabnet.datasus.gov.br

30. Oitavo Distrito do Instituto de Meteorologia. Quadro da temperatura média mensal nos anos de 2001 a 2005. [Consulta pelo e-mail: seoma8@inmet.gov.br]

31. Lasmar L, Goulart E, Sakurai E, Camargos P. Fatores de risco para hospitalização de crianças e adolescentes asmáticos. Revista de Saúde Pública. 2002; 36(4): 409-19. 32. Barros AJ, Hirata VN. Alternatives for logistic regression in cross-sectional studie comparison of models that directly estimative the prevalence rat. BMC Med Res Methodol. Oct. 2003; 20(1): 21-3.

33. Castro MSM. A utilização das internações hospitalares no Brasil: fatores associados, grandes usuários, reinternações e efeito de oferta de serviços sobre o uso. [Tese]. Rio de Janeiro: Escola Nacional de Saúde Pública, Fundação Oswaldo Cruz. Disponível em: http://bases.bireme.br/ cgi-bin/wxis/ind.exe/iah/online. 


\section{Endereço para correspondência:}

Núcleo de Epidemiologia do Serviço de Saúde Comunitária

do Hospital Nossa Senhora da Conceição.

Rua Francisco Trein, 596

Porto Alegre - RS

CEP: 91350-200

\section{Endereço eletrônico:}

mlenz@terra.com.br 